\title{
Experiencia de 18 años de cirugía de obesidad en la Pontificia Universidad Católica de Chile
}

\author{
SERGIO GUZMÁN ${ }^{1}$, MÓNICA MANRIQUE², ALEJANDRO RADDATZ', \\ ENRIQUE NORERO' ${ }^{1}$, JOSÉ SALINAS ${ }^{a}$, PABLO ACHURRA ${ }^{\text {b }}$ \\ RICARDO FUNKE ${ }^{1}$, CAMILO BOZA ${ }^{1}$, FERNANDO CROVARI ${ }^{1}$, \\ ALEX ESCALONA ${ }^{1}$, GUSTAVO PÉREZ ${ }^{1}$, FERNANDO PIMENTEL ${ }^{1}$, \\ JULIETTA KLASSEN ${ }^{2}$, LUIS IBÁÑEZ ${ }^{1}$
}

\section{Results of bariatric surgery. Experience over 18 years}

\begin{abstract}
Background: Bariatric surgery is the gold-standard treatment for morbid obesity because it has low morbidity rates in high-volume centers and generates long term sustained weight loss. Aim: To describe our experience in bariatric surgery since the creation of our bariatric program in 1992. Material and Methods: Retrospective analysis of all patients subjected to bariatric surgery from 1992 to December 2010. Data was obtained from the electronic institutional registry. The Procedures performed were open and laparoscopic Roux-en-Y gastric bypass (BPGA and BPGL, respectively), laparoscopic adjustable gastric band (BGAL) and laparoscopic sleeve gastrectomy (GML). Results: A total of 4943 procedures were performed, 768 (16\%) BPGA, 2558 (52\%) BPGL, 199 (4\%) BGAL and 1418 (29\%) GML. The number of procedures progressively increased, from 100 cases in 2000 to over 700 cases in 2008. Proportion of females and preoperative mean body mass index fluctuated between 69 and $79 \%$ and 35 and $43 \mathrm{~kg} / \mathrm{m}^{2}$, respectively, among the different procedures. Early and late complications fluctuated between 0 to 1\% (higher on BPGA) and 3 to $32.7 \%$ (higher on $B G A L$ ), respectively. The excess weight lost at five years was $76.1 \%$ in BPGA, 92.5\% in BPGL and 53.7\% in BGAL. The figure for GML at three years was $73.7 \%$. Conclusions: The complication rates of this series of patients are similar to those reported in large series abroad. BPGL is still the most effective procedure; however GML is an attractive alternative for less obese patients.
\end{abstract}

(Rev Med Chile 2013; 141: 553-561).

Key words: Bariatric surgery; Gastric bypass; Obesity, morbid.

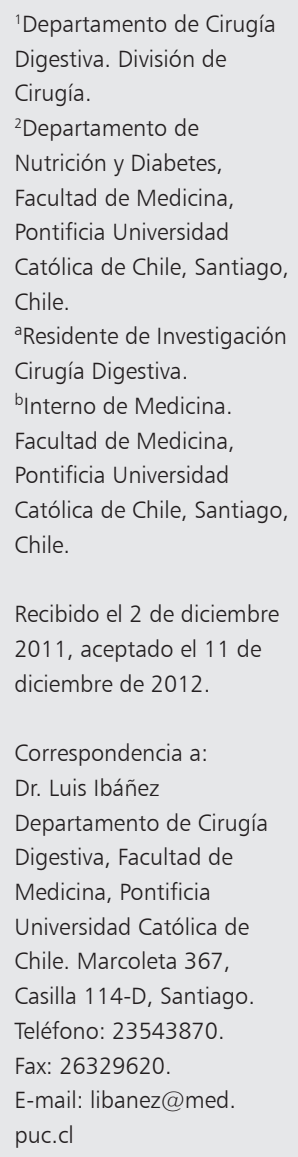

Recibido el 2 de diciembre 2011, aceptado el 11 de diciembre de 2012.

Correspondencia a: Dr. Luis Ibáñez Departamento de Cirugía Digestiva, Facultad de Medicina, Pontificia Universidad Católica de Chile. Marcoleta 367, Casilla 114-D, Santiago. Teléfono: 23543870. Fax: 26329620. E-mail: libanez@med. puc.cl

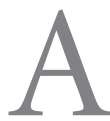
ctualmente la obesidad se ha transformado en una epidemia mundial ${ }^{1}$. En Chile, la obesidad afecta a $22 \%$ de la población y la obesidad mórbida a $1,3 \%{ }^{2}$. La terapia con dieta y la farmacoterapia han fracasado en lograr un tratamiento efectivo ${ }^{3,4}$. Actualmente la cirugía bariátrica es la única alternativa que ha demostrado una baja de peso y resolución de comorbilidades significativa a largo plazo $^{5-7}$.

En 1992, el Departamento de Cirugía Digestiva en conjunto con el Departamento de Nutrición y Diabetes de la Facultad de Medicina de la Pontificia Universidad Católica de Chile, se implementó un programa para el tratamiento quirúrgico de la obesidad, se seleccionó el bypass gástrico en Y-deRoux por vía abierta (BPGA) como técnica inicial. Progresivamente se fue consolidando un programa multidisciplinario. Posteriormente, se desarrolló el bypass gástrico por vía laparoscópica $(\mathrm{BPGL})^{8-12}$. Este abordaje produjo un aumento significativo de 
la cirugía bariátrica y constituye en la actualidad la vía de elección de esta técnica. En años más recientes se incorporaron la banda gástrica ajustable laparoscópica (BGAL) y la gastrectomía en manga laparoscópica (GML).

El objetivo de este trabajo es describir la experiencia y resultados de este programa para el tratamiento quirúrgico de la obesidad desde sus inicios.

\section{Material y Métodos}

Análisis retrospectivo de todos los pacientes sometidos a cirugía bariátrica operados desde 1992 hasta diciembre de 2010. Los datos se obtuvieron del registro prospectivo electrónico institucional.

Desde sus inicios el tratamiento quirúrgico de la obesidad fue concebido como un programa multidisciplinario, el cual se ha ido potenciado y perfeccionando a lo largo de los años. Todo paciente es evaluado por un equipo de cirujanos digestivos, nutriólogos, nutricionistas, psicólogos y kinesiólogos. Este equipo evalúa las comorbilidades y entrega una completa educación acerca del programa. Luego el paciente es reevaluado por el cirujano bariátrico el cual recomienda la técnica quirúrgica más adecuada. Se solicita de rutina a los pacientes una baja de peso preoperatoria, de $5-10 \%$, indicación que se ha hecho cada vez más estricta a lo largo del tiempo. Una vez que el paciente completa su evaluación, ingresa a la base de datos prospectiva. La selección de pacientes se realizó según las guías propuestas por National Institutes of Health $(\mathrm{NIH})$ para cirugía bariátrica ${ }^{13}$ en la mayoría de los casos (Figura 1).

\section{Técnica quirúrgica}

La primera técnica empleada fue el BPGA. En un comienzo la bolsa gástrica se confeccionó en forma transversal y sin sección, para luego hacerla vertical y con sección, disminuyendo su tamaño hasta un volumen de 10-15 ml. La anastomosis gastroyeyunal se realizó de $1 \mathrm{~cm}$ de diámetro con sutura manual en un plano, con vicryl ${ }^{\circledR}$ 3-0. La Y-de-Roux se realiza con una rama alimentaria de 150-200 cm, ascendida por vía transmesocólica en la vía abierta.

$\mathrm{Al}$ incorporar el BPGL, se seleccionó la técnica que más se asemejaba al abordaje por laparotomía, confeccionando una bolsa pequeña, de 15-20 ml, calibrada con una sonda de 34F. La anastomosis gastroyeyunal se realiza en forma manual en dos planos con vicryl ${ }^{\circledR} 3-0$. La Y-de-Roux es de 150$200 \mathrm{~cm}$, ascendida por vía transmesocólica hasta el año 2003 y antecólica desde el 2004. Se utilizó seda 2-0 para el cierre de las brechas mesentéricas y apertura del mesocolon transverso, y para el cierre del espacio de Petersen en la técnica laparoscópica.

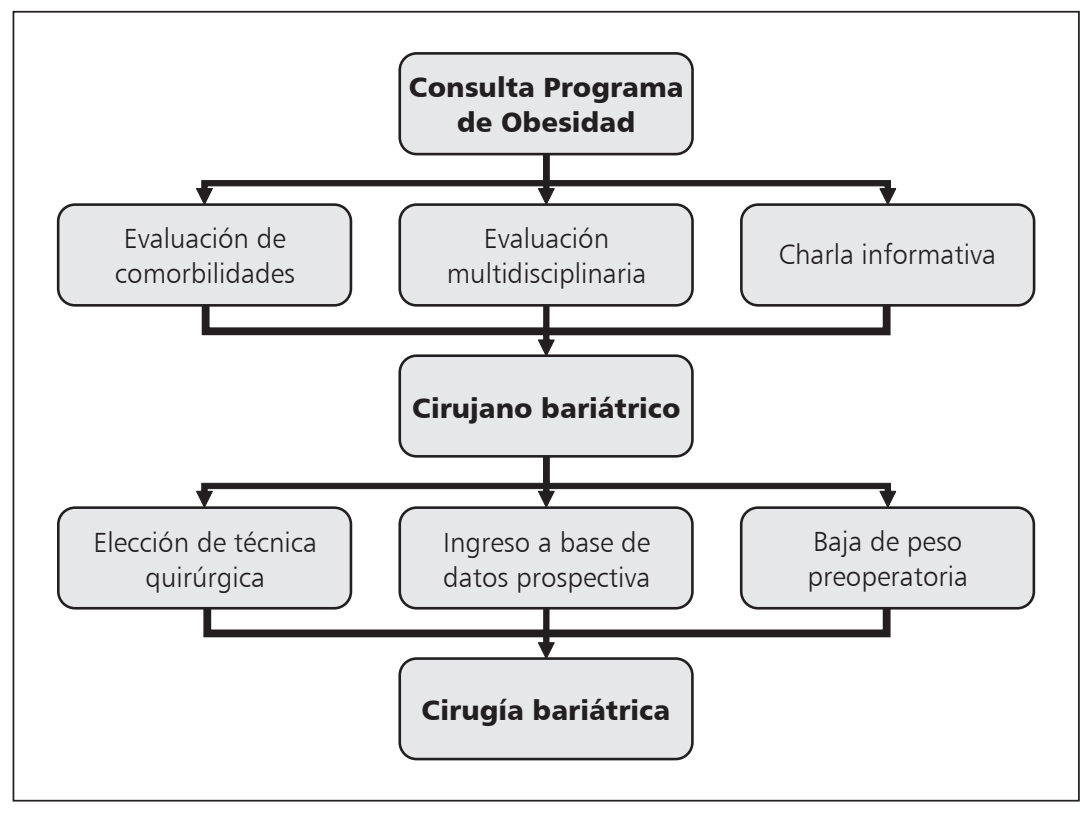

Figura 1. Evaluación y manejo preoperatorio de pacientes sometidos a cirugía de obesidad. 
Para las BGAL se utilizó la técnica de disección retrogástrica a través de la pars fláccida. Se utilizaron bandas Lap-Band ${ }^{\circledR}$ y Johnson\&Johnson ${ }^{\circledR}$. Se confeccionó un túnel laxo alrededor de la banda, consistente en pared anterior gástrica y fondo, suturado al reservorio gástrico proximal con ethibond 2-0. El catéter se extrae por el sitio de inserción del trocar subcostal izquierdo y se conecta al reservorio, que luego se fija en un bolsillo supra-aponeurótico.

La GML consiste en la resección longitudinal del cuerpo gástrico y el fondo, calibrando el estómago remanente en torno a una sonda inicialmente de 60 French, y desde fines de 2009 con sonda de 34 ó 50 French. Se utiliza refuerzo de la línea de corchetes con sutura continua, utilizando material absorbible. El estómago resecado se extrae por la ampliación del puerto del trocar en flanco izquierdo dentro de una bolsa.

En ninguno de los procedimientos se realiza control postoperatorio radiológico de rutina, ni se dejan drenajes.

Posterior a la cirugía, los pacientes son trasladados a recuperación por dos horas y luego a sala, manteniéndose con compresión neumática intermitente de extremidades las primeras 24 h. Se inicia heparina de bajo peso molecular en dosis profilácticas desde el primer día postoperatorio. Todos los pacientes reciben inhibidores de la bomba de protones. Se inicia una dieta líquida fraccionada al día siguiente de la operación en el caso del BPGL, BGAL o GML, y subsiguiente en el caso del BPGA. Esta se mantiene por una semana en BPGA, BPGL y GML, y por tres días en la BGAL. Luego se indica un régimen papilla durante un mes postoperatorio, para luego cambiar a régimen picado y en forma progresiva, dependiendo de la tolerancia individual, se indica una alimentación de consistencia normal. Se realizan controles postoperatorios a los 10 días, 1, 3, 6 y 12 meses, para luego continuar con controles anuales. Se realiza suplementación mediante polivitamínicos, complejos de vitamina B intramuscular, calcio y fierro. En pacientes con BGAL se realiza un primer ajuste a las seis semanas, y posteriormente según evolución.

Se consideró como complicación precoz a aquella que se produjo dentro de los primeros treinta días del postoperatorio, y tardía a aquella que ocurrió con posterioridad a este período. La mortalidad quirúrgica se consideró como la muerte por cualquier causa ocurrida dentro de los treinta días del postoperatorio o durante la hospitalización. Se utilizaron los test de $\chi^{2}$ y t de Student para comparar los resultados perioperatorios y las complicaciones de los 4 procedimientos.

Se actualizó el seguimiento de los primeros 1.500 BPGL hasta diciembre de 2007, las primeras 1.000 GML hasta febrero de 2010, todas las BGAL hasta mayo de 2009. Se realizó el seguimiento hasta 5 años. Se calculó el índice de masa corporal (IMC), la pérdida del exceso de peso (PEP) y el éxito del procedimiento (PEP $\geq 50 \%$ ).

\section{Resultados}

Desde el año 1992 hasta diciembre de 2010, se realizaron 4.943 intervenciones quirúrgicas, incluyendo 768 BPGA, 2.558 BPGL, 199 BGAL y 1.418 GML.

En 1992 se inició nuestra experiencia con el BPGA como técnica quirúrgica única. En el año 2001 se realizaron los primeros BPGL, llegando a igualar en número al BPGA hacia el 2003, para luego desplazarlo casi por completo en la actualidad. En el año 2002 se inició la experiencia con la BGAL, llegando a su máxima expresión el año 2003, para luego caer en desuso hacia el año 2007. A principios de 2005 se incorporó la GML, la cual experimentó un veloz incremento, hasta llegar a igualar al BPGL (Figura 2). El volumen quirúrgico llegó a los 100 procedimientos anuales hacia el año 2000, y continuó aumentando progresivamente hasta llegar a más de 700 casos el año 2008.

La distribución por sexo fue similar en todas las técnicas. La edad promedio también fue similar entre las técnicas, excepto en el BPGA, que presentó una edad promedio mayor $(\mathrm{p}<0,0001)$. El IMC preoperatorio fue mayor en el BPGA, comparado con los otros 3 procedimientos $(\mathrm{p}<0,0001)$ (Tabla 1).

Los tiempos operatorios fueron mayores en el BPGA y BPGL, con un promedio de $112 \mathrm{~min}$. La BGAL fue la que presentó el menor tiempo operatorio $(\mathrm{p}<0,0001)$. El tiempo de hospitalización del BPGA fue significativamente mayor que en el BPGL ( $p<0,0001)$. A su vez, los tiempos de hospitalización de la BGAL y GML fueron significativamente menores al bypass $(\mathrm{p}<0,0001)$ (Tabla 1$)$.

La conversión a técnica abierta fue necesaria en $1 \%$ de los BPGL y en $0,1 \%$ de las GML. No hubo conversión para BGAL. 


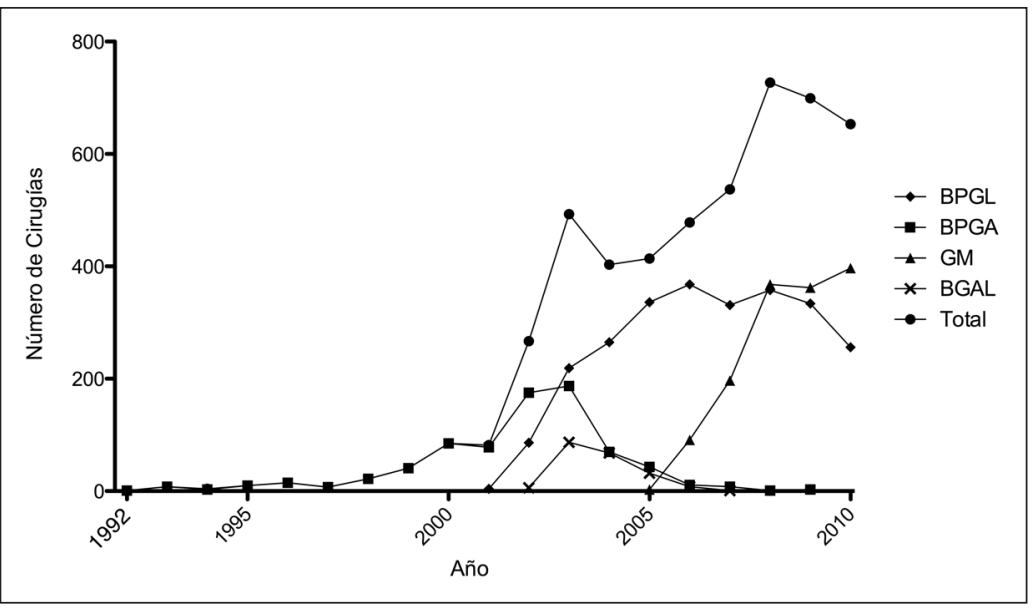

Figura 2. Actividad total y según procedimiento específico de cirugía de obesidad entre 1992 y 2010.

Tabla 1. Datos demográficos, índice de masa corporal preoperatorios y resultados de la cirugía

\begin{tabular}{|lcccc|}
\hline Procedimiento & BPGA & BPGL & BGAL & GML \\
\hline N & 768 & 2.558 & 199 & 1.418 \\
\hline Sexo femenino (\%) & $69 \%$ & $73 \%$ & $70 \%$ & $79 \%$ \\
\hline Edad (años) & $39 \pm 11$ & $37 \pm 11$ & $37 \pm 12$ & $36 \pm 11$ \\
IMC (kg/m²) & $43 \pm 6$ & $39 \pm 5$ & $36 \pm 4$ & $35 \pm 4$ \\
\hline Tiempo operatorio (min) & $112 \pm 40$ & $112 \pm 45$ & $70 \pm 22$ & $76 \pm 27$ \\
Duración hospitalización (días) & $9 \pm 12,7$ & $3,6 \pm 5,9$ & $1,8 \pm 0,8$ & $2,9 \pm 1,7$ \\
\hline Conversión a laparotomía (\%) & na & $1 \%$ & $0 \%$ & $0,1 \%$ \\
\hline Complicación precoz (\%) & $11 \%$ & $7 \%$ & $0,5 \%$ & $3 \%$ \\
\hline Complicación tardía (\%) & $27 \%$ & $14 \%$ & $34 \%$ & $3 \%$ \\
Complicaciones totales (\%) & $38 \%$ & $19 \%$ & $34 \%$ & $6 \%$ \\
\hline Reoperación (\%) & $2,3 \%$ & $1,3 \%$ & $0,5 \%$ & $0,3 \%$ \\
\hline Mortalidad operatoria. & $0,3 \%$ & $0 \%$ & $0 \%$ & $0 \%$ \\
\hline
\end{tabular}

BPGA: bypass gástrico abierto. BPGL: bypass gástrico laparoscópico. BGAL: banda gástrica ajustable laparoscópica. GML: gastrectomía en manga laparoscópica. IMC: índice de masa corporal. NA: No se aplica. ${ }^{\dagger}$ Reoperación durante los primeros 30 días de postoperado.

\section{Complicaciones}

El BPGA tuvo 11\% de complicaciones menores, diferencia que es significativamente mayor que el BPGL (7\%) ( $\mathrm{p}<0,0001)$. La GML y la BGAL tuvieron tasas de complicaciones precoces de 3 y $0,5 \%$ respectivamente, significativamente menores al bypass gástrico $(\mathrm{p}<0,0001)$. Las complicaciones tardías se observaron en 27\% de los BPGA, reduciéndose a $14 \%$ con el abordaje laparoscópico (p $<0,0001)$. La GML presentó la menor incidencia de complicaciones tardías comparado con las otras técnicas $(p<0,0001)$. En el otro extremo la BGAL fue la técnica con mayor morbilidad a largo plazo $(\mathrm{p}<0,0001)$.

En toda la serie se registraron $2(0,04 \%)$ pacientes fallecidos, que se produjeron en el grupo de BPGA, debido a una filtración de la anastomosis yeyuno-yeyunal en la base de la Y-de-Roux y a un tromboembolismo pulmonar masivo al segundo día postoperatorio.

En la Tabla 2 se describen las complicaciones según técnica quirúrgica. No hubo diferencia en la incidencia de filtraciones (bolsa gástrica o anastomosis gastroyeyunal) y estenosis entre el 
Resultados en cirugía bariátrica - S. Guzmán et al

Tabla 2. Complicaciones específicas según procedimiento

\begin{tabular}{|c|c|c|c|c|}
\hline Procedimiento & BPGA & BPGL & BGAL & GML \\
\hline Filtración* & $1,5 \%$ & $1 \%$ & na & 0,4 \\
\hline Hemorragia digestiva & $1,9 \%$ & $1,5 \%$ & $0,5 \%$ & $0,1 \%$ \\
\hline Hemoperitoneo & $0,2 \%$ & $0,2 \%$ & $0,5 \%$ & $0,7 \%$ \\
\hline Enfermedad tromboembólica** & $0,4 \%$ & $0,4 \%$ & $0 \%$ & $0,1 \%$ \\
\hline Trombosis portal/mesentérica & $0 \%$ & $0,04 \%$ & $0 \%$ & $0,8 \%$ \\
\hline Colección intrabdominal & $0,5 \%$ & $0,4 \%$ & $0 \%$ & $0,1 \%$ \\
\hline Complicaciones de la herida ${ }^{\dagger}$ & $3,8 \%$ & $0,4 \%$ & $1 \%$ & $0,2 \%$ \\
\hline Respiratorias $^{\dagger \dagger}$ & $2 \%$ & $0,4 \%$ & $0 \%$ & $0,1 \%$ \\
\hline Estenosis GY & $5,1 \%$ & $5,4 \%$ & na & na \\
\hline íleo mecánico & $4,2 \%$ & $4,4 \%$ & $0,5 \%$ & $0 \%$ \\
\hline Biliares $^{\ddagger}$ & $5 \%$ & $4,7 \%$ & $3,5 \%$ & $2,1 \%$ \\
\hline Hernia incisional & $9,1 \%$ & $0,8 \%$ & $0,5 \%$ & $0,2 \%$ \\
\hline
\end{tabular}

BPGA: bypass gástrico abierto. BPGL: bypass gástrico laparoscópico. BGAL: banda gástrica ajustable laparoscópica. GML: gastrectomía en manga laparoscópica. GY: gastro-yeyuno anastomosis de bypass gástrico. *Filtración de la bolsa gástrica, anastomosis gastro-yeyunal o de línea de sección de GML. ${ }^{* *}$ Considera trombosis venosa profunda o tromboembolismo pumonar. ${ }^{\dagger}$ Considera seroma o infección de la herida operatoria. ${ }^{\dagger \dagger}$ Considera atelectasia o neumonía nosocomial. ${ }^{*}$ Considera colelitiasis, coledocolitiasis o pancreatitis aguda.

bypass gástrico vía abierta o laparoscópica. Todas las estenosis fueron tratadas exitosamente con dilataciones endoscópicas. La GML tuvo menor incidencia de filtraciones que el BPGL que no fue estadísticamente significativa $(0,4$ vs $1 \%$ respectivamente, $\mathrm{p}=\mathrm{ns}$ ). La frecuencia de enfermedad tromboembólica fue similar en todos los procedimientos excepto en la BGAL que no presentó esta complicación.

Las técnicas efectuadas por vía laparoscópica presentaron significativamente menos complicaciones de la herida, complicaciones respiratorias y hernias incisionales al ser comparadas con el BPGA.

El hemoperitoneo fue significativamente más frecuente en los pacientes sometidos a GML $(0,7 \%)$ en comparación con el bypass gástrico $(0,2 \%)(\mathrm{p}=0,017)$. La trombosis portomesentérica se presentó en $1(0,04 \%)$ caso de BPGL, y en 11 $(0,8 \%)$ pacientes sometidos a GML $(\mathrm{p}<0,0001)$.

La hemorragia digestiva, el íleo mecánico y las complicaciones biliares fueron complicaciones más frecuentes en los pacientes sometidos a bypass gástrico, al compararlos con la BGAL y la GML $(\mathrm{p}<0,0001)$. No existió diferencia para estas complicaciones entre bypass gástrico abierto o laparoscópico.

Las complicaciones más frecuentes de la BGAL fueron la intolerancia al dispositivo, el deslizamiento y la disfunción del reservorio. Veinte por ciento de los pacientes necesitaron una reoperación alejada por alguna complicación y $14 \%$ de las bandas debieron ser removidas por diferentes causas, de estos casos $71 \%$ se convirtió a BPGL o GML (Tabla 3).

La PEP a un año fue de 91\% para BPGL, $82 \%$ para BPGA, $58 \%$ para BGAL y $86 \%$ para GML (Tabla 4). En el seguimiento más alejado, la PEP del BPGL bajó a 85\% a 3 años y luego aumentó a $92 \%$ a 5 años. Para BGAL el promedio de PEP se mantuvo entre 59-56\% hasta los 5 años, pero con una desviación estándar en aumento, con un amplio rango de resultados y una caída en el éxito del procedimiento a sólo 53\%. Para GML sólo existen resultados a 3 años, pero el comportamiento de la GML resultó similar al obtenido con el BPGL con una PEP de $93 \%$ a 3 años. La evolución de la PEP e IMC en el tiempo están graficados en las Figuras 3 y 4 respectivamente. 


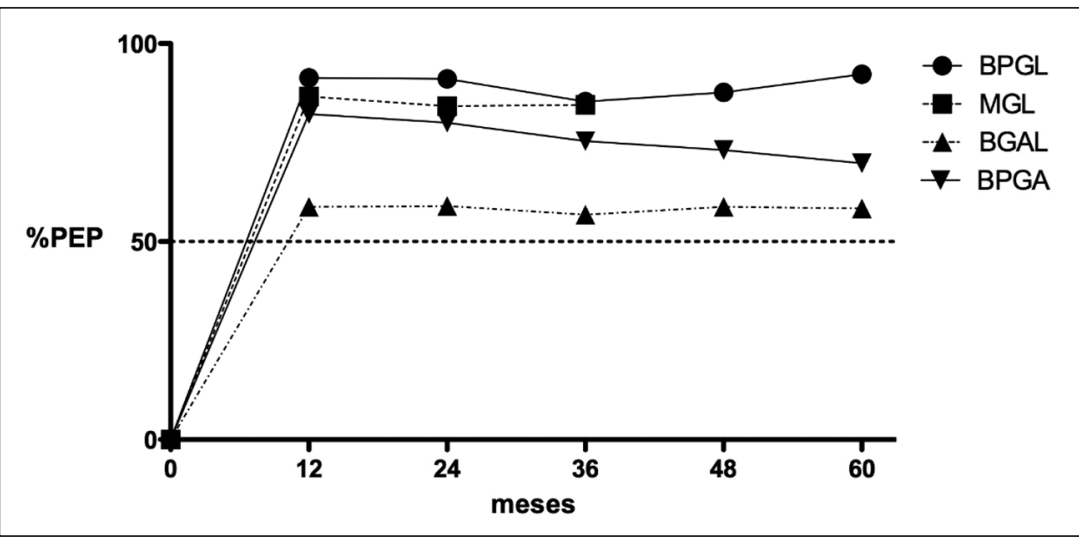

Figura 3. Porcentaje de pérdida del exceso de peso con las distintas técnicas.

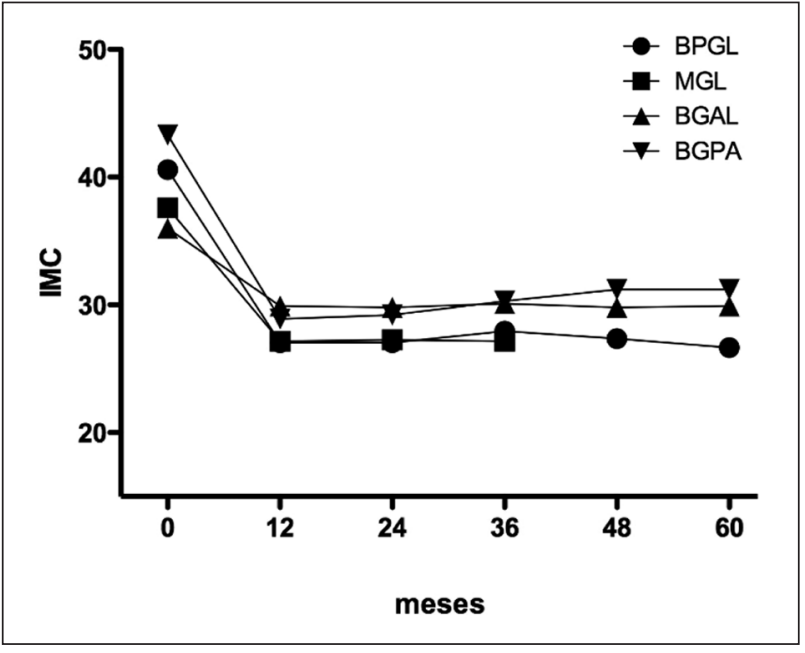

Figura 4. Índice de masa corporal en el seguimiento de las distintas técnicas.
Tabla 3. Complicaciones tardías de banda gástrica ajustable laparoscópica

\begin{tabular}{|lc|}
\hline Complicación & (\%) \\
\hline Relacionadas a la banda & $18 \%$ \\
\hline Intolerancia alimentaria & $6,5 \%$ \\
\hline Deslizamiento de banda & $6 \%$ \\
Disfunción del reservorio & $4 \%$ \\
\hline Erosión de banda & $0,5 \%$ \\
Ileo mecánico & $0,5 \%$ \\
Infección del reservorio & $0,5 \%$ \\
No relacionadas a la banda & $16 \%$ \\
Anemia & $7,5 \%$ \\
Alopecia & $4,5 \%$ \\
Total & $3,5 \%$ \\
\hline
\end{tabular}

\section{Discusión}

La obesidad es una patología que afecta en forma importante la calidad y expectativas de vida de las personas. Un IMC $\geq 30 \mathrm{~kg} / \mathrm{m}^{2}$ aumenta en $70 \%$ el riego de muerte respecto a la población normal $^{18}$, y reduce en $22 \%$ las expectativas de vida ${ }^{19}$. A pesar de ser un problema de salud pública tan evidente $^{20}$, sólo los últimos diez años se ha generado un reconocimiento de esta epidemia. Desde hace más de 50 años que existe la cirugía bariátrica, pero sólo en 1991 se realiza el primer esfuerzo por crear normas y regular su indicación ${ }^{21}$.

En Chile la publicación de los primeros casos de cirugía bariátrica se remonta a 1986, cuando
González y cols. describieron seis bypass yeyunoileales. Posteriormente, otros grupos nacionales se sumaron a esta nueva área de la cirugía digestiva, incluyendo al nuestro, efectuando el primer bypass gástrico en $1992^{22-26}$. En los últimos diez años el número de procedimientos bariátricos ha aumentado drásticamente en todo el mundo ${ }^{27}$, incluyendo nuestro país ${ }^{28}$. Esta serie, que incluye la totalidad de la cirugía de obesidad realizada por un centro, refleja esta tendencia.

Al tomar la decisión de iniciar el tratamiento quirúrgico de la obesidad en nuestro centro, se estudiaron las diferentes técnicas disponibles seleccionando al BPGA como técnica inicial. Esto debido a la consistencia de los buenos resultados 
Resultados en cirugía bariátrica - S. Guzmán et al

Tabla 4. Evaluación de seguimiento, pérdida del exceso de peso y éxito del procedimiento para bypass gástrico laparoscópico (A), bypass gástrico abierto (B), banda gástrica ajustable laparoscópica (C) y gastrectomía en manga laparoscópica (D)

\begin{tabular}{|c|c|c|c|c|c|}
\hline Años & 1 & 2 & 3 & 4 & 5 \\
\hline \multicolumn{6}{|c|}{ (A) Bypass gástrico laparoscópico } \\
\hline $\mathrm{IMC}\left(\mathrm{kg} / \mathrm{mt}^{2}\right)$ & $27,0 \pm 4,1$ & $27,0 \pm 4,1$ & $27,9 \pm 4,5$ & $27,3 \pm 4,1$ & $26,6 \pm 4,2$ \\
\hline PEP (\%) & $91,4 \pm 24,1$ & $91,0 \pm 24,9$ & $85,3 \pm 25,7$ & $87,7 \pm 25,0$ & $92,2 \pm 26,1$ \\
\hline Éxito (\%) & 97,1 & 97,0 & 93,1 & 88,5 & 92,5 \\
\hline \multicolumn{6}{|c|}{ *IMC Basal 40,5 \pm 5,2 } \\
\hline \multicolumn{6}{|c|}{ (B) Bypass gástrico abierto } \\
\hline $\mathrm{IMC}\left(\mathrm{kg} / \mathrm{mt}^{2}\right)$ & $28,9 \pm 5,5$ & $29,2 \pm 5,3$ & $30,3 \pm 6,1$ & $31,2 \pm 6,6$ & $31,2 \pm 5,6$ \\
\hline PEP (\%) & $82,2 \pm 29,7$ & $80,0 \pm 24,6$ & $75,4 \pm 26,5$ & $73,1 \pm 25,7$ & $69,8 \pm 26,1$ \\
\hline Éxito (\%) & 90,8 & 90,3 & 84,1 & 84,2 & 76,1 \\
\hline \multicolumn{6}{|c|}{ *IMC Basal 43,3 $\pm 6,8$} \\
\hline \multicolumn{6}{|c|}{ (C) Banda gástrica ajustable laparoscópica } \\
\hline $\mathrm{IMC}\left(\mathrm{kg} / \mathrm{mt}^{2}\right)$ & $29,9 \pm 3,9$ & $29,8 \pm 3,9$ & $30,1 \pm 4,4$ & $29,8 \pm 4,6$ & $29,9 \pm 5,4$ \\
\hline PEP (\%) & $58,8 \pm 30,0$ & $59,0 \pm 34,1$ & $56,8 \pm 35,0$ & $58,8 \pm 38,8$ & $58,4 \pm 46,6$ \\
\hline Éxito (\%) & 59,6 & 58,2 & 56,5 & 60,7 & 53,7 \\
\hline \multicolumn{6}{|c|}{ *IMC basal 36,0 $\pm 3,8$} \\
\hline \multicolumn{6}{|c|}{ (D) Gastrectomía en manga laparoscópica } \\
\hline $\mathrm{IMC}\left(\mathrm{kg} / \mathrm{m}^{2}\right)$ & $27,1 \pm 3,5$ & $27,7 \pm 3,6$ & $27,1 \pm 3,4$ & & \\
\hline PEP (\%) & $86,7 \pm 26,8$ & $84,2 \pm 28,5$ & $84,5 \pm 27,8$ & & \\
\hline Éxito (\%) & 92,2 & 90,0 & 93,7 & & \\
\hline \multicolumn{6}{|c|}{ IMC basal 37,4 $\pm 4,0$} \\
\hline
\end{tabular}

en cuanto a la baja de peso y menor morbilidad, en comparación a otros procedimientos con mayor componente malabsortivo ${ }^{14-17}$. Posteriormente, y en asociación con un entrenamiento en laparoscopia avanzada, se introdujo el BPGL el 2001, realizando la misma cirugía pero con abordaje mínimamente invasivo. Esto produjo una disminución significativa en las complicaciones postoperatorias, en especial la morbilidad respiratoria, las complicaciones de la herida y las hernias incisionales en el largo plazo. La búsqueda de alternativas menos invasivas para pacientes menos obesos y con menos comorbilidades, ha llevado a la introducción primero de la BGAL en 2002 y luego de la GML en 2005. La BGAL fue abandonada el año 2006 debido al menor porcentaje de éxito comparado con el BPGL y la GML, la necesidad de un control y ajustes de la banda más estrictos.

Al comparar los resultados de las tres técnicas quirúrgicas bariátricas que actualmente se realizan con mayor frecuencia por vía laparoscópica, podemos observar que a pesar de no ser comparables por IMC, el grupo de bypass respecto a la
BGAL y GML, logra un mayor PEP a mediano plazo. Sin embargo, la morbilidad precoz y tardía sigue siendo mayor en el bypass que en la GML. Es interesante observar que los resultados de PEP de la GML se acercan notablemente a los del bypass y son significativamente superiores a los de la BGAL. $\mathrm{El}$ argumento más frecuente para preferir la BGAL por sobre la GML, es que es un procedimiento más sencillo y con menor morbilidad. Sin embargo, esto se cumple sólo en el corto plazo, ya que al analizar las complicaciones tardías y la necesidad de reoperaciones, estas son mayores en el grupo de la BGAL. Por otro lado, la estadía hospitalaria y necesidad de conversión a cirugía abierta en la GML no difieren en forma significativa de la BGAL.

En los últimos 15 años, hemos logrado establecer un programa de tratamiento quirúrgico de la obesidad, con excelentes resultados a largo plazo, con una tasa de complicaciones comparables con las grandes series publicadas en la literatura mundial ${ }^{29-35}$. En nuestra experiencia, la mortalidad global postoperatoria es de $0,04 \%$. Pensamos que para obtener estos resultados, además de un alto 
volumen quirúrgico, es necesaria una revisión y discusión constante de las normas de trabajo, lo cual permite realizar cambios que logren disminuir el número de complicaciones. Este es el caso del cambio de técnica que realizamos con el BPGL en el 2004, al comenzar a ascender el asa alimentaria por vía antecólica, disminuyendo diez veces la incidencia de íleo mecánico, y eliminando las obstrucciones intestinales en el postoperatorio precoz ${ }^{36}$. Un alto volumen quirúrgico permite reducir el número de complicaciones y su mejor tratamiento ${ }^{37}$, ya sea en nuestros propios pacientes o en aquellos derivados de otros centros, al establecer hábitos y protocolos de trabajo. Es el caso del manejo de las estenosis gastroyeyunales, mediante dilatadores de Savary ${ }^{38}$, y más recientemente el uso de endoprótesis esofágicas, para filtraciones en BPYR y GM.

En conclusión podemos decir que es posible realizar en nuestro medio un programa multidisciplinario de cirugía bariátrica de alto volumen quirúrgico, con buenos resultados perioperatorios inmediatos y a largo plazo. El bypass gástrico en $\mathrm{Y}$ de Roux sigue siendo el procedimiento más efectivo para el tratamiento de la obesidad mórbida en nuestro medio.

\section{Referencias}

1. Haslam DW, James WP. Obesity. Lancet 2005; 366: 1197 209.

2. Minsal 2003, Primera Encuesta Nacional de Salud. Gobierno de Chile, Ministerio de Salud.

3. Buchwald H, Avidor Y, Braunwald E, Jensen MD, Pories W, Fahrbach K, et al. Bariatric surgery: a systematic review and meta-analysis. Jama 2004; 292: 1724-37.

4. Carrasco F, Klaassen J, Papapietro K, Reyes E, Rodríguez L, Csendes A, et al. [A proposal of guidelines for surgical management of obesity]. Rev Med Chile 2005; 133: 699706.

5. Sjostrom CD, Lissner L, Wedel H, Sjostrom L. Reduction in incidence of diabetes, hypertension and lipid disturbances after intentional weight loss induced by bariatric surgery: the SOS Intervention Study. Obes Res 1999; 7: 477-84.

6. Maggard MA, Shugarman LR, Suttorp M, Maglione M, Sugerman HJ, Livingston EH, et al. Meta-analysis: surgical treatment of obesity. Ann Intern Med 2005; 142: 547-59.

7. Buchwald H. The future of bariatric surgery. Obes Surg 2005; 15: 598-605.
8. Wittgrove AC, Clark GW, Tremblay LJ. Laparoscopic Gastric Bypass, Roux-en-Y: Preliminary Report of Five Cases. Obes Surg 1994; 4: 353-7.

9. Wittgrove AC, Clark GW. Laparoscopic gastric bypass, Roux-en-Y- 500 patients: technique and results, with 3-60 month follow-up. Obes Surg 2000; 10: 233-9.

10. Higa KD, Boone KB, Ho T, Davies OG. Laparoscopic Roux-en-Y gastric bypass for morbid obesity: technique and preliminary results of our first 400 patients. Arch Surg 2000; 135: 1029-34.

11. Higa KD, Ho T, Boone KB. Laparoscopic Roux-en-Y gastric bypass: technique and 3-year follow-up. J Laparoendosc Adv Surg Tech A 2001; 11:377-82.

12. Higa KD, Boone KB, Ho T. Complications of the laparoscopic Roux-en-Y gastric bypass: 1,040 patients--what have we learned? Obes Surg 2000; 10: 509-13.

13. Hubbard VS, Hall WH. Gastrointestinal Surgery for Severe Obesity. Obes Surg 1991; 1: 257-65.

14. Parikh MS, Laker S, Weiner M, Hajiseyedjavadi O, Ren CJ. Objective comparison of complications resulting from laparoscopic bariatric procedures. J Am Coll Surg 2006; 202: 252-61.

15. Fisher BL, Schauer P. Medical and surgical options in the treatment of severe obesity. Am J Surg 2002; 184: 9S-16S.

16. Gracia JA, Martínez M, Aguilella V, Elia M, Royo P. Postoperative morbidity of biliopancreatic diversion depending on common limb length. Obes Surg 2007; 17: 1306-11.

17. Bloomberg RD, Fleishman A, Nalle JE, Herron DM, Kini S. Nutritional deficiencies following bariatric surgery: what have we learned? Obes Surg 2005; 15: 145-54.

18. Peeters A, Barendregt JJ, Willekens F, Mackenbach JP, Al Mamun A, Bonneux L. Obesity in adulthood and its consequences for life expectancy: a life-table analysis. Ann Intern Med 2003; 138: 24-32.

19. Fontaine KR, Redden DT, Wang C, Westfall AO, Allison DB. Years of life lost due to obesity. Jama JAMA 2003; 289: 187-93.

20. Obesity: preventing and managing the global epidemic. Report of a WHO consultation. World Health Organ Tech Rep Ser 2000; 894: i-xii, 1-253.

21. NIH conference. Gastrointestinal surgery for severe obesity. Consensus Development Conference Panel. Ann Intern Med 1991; 115: 956-61.

22. González R, Rossi G, González F, López MI, Fischer S. Obesidad mórbida: experiencia con el bypass yeyuno ileal en 6 pacientes. Rev Med Chile 1986; 114: 29-35.

23. Awad W, Loehnert R, Jirón R, Jirón A, Stambuk J. Obesidad mórbida. Tratamiento quirúrgico; gastroplastía. Rev Chil Cir 1993; 45: 407-12.

24. Awad W, Loehnert R, Gastroplastía laparoscópica. Técni- 
ca y resultados preliminares con pacientes con obesidad mórbida. Rev Chil Cir 1997; 49: 64-68.

25. Csendes A, Burdiles P, Jensen C, Díaz JC, Cortés C, Rojas J, et al. Resultados preliminares de la gastroplastía horizontal con anastomosis en Y-de-Roux como cirugía baríatrica en pacientes con obesidad severa y mórbida. Rev Med Chile 1999; 127: 953-60.

26. Guzmán S, Manríque M. Tratamiento quirúrgico de la obesidad mórbida. Cuad Cir 1999; 13: 24-8.

27. Buchwald H, Williams SE. Bariatric surgery worldwide 2003. Obes Surg 2004; 14: 1157-64.

28. Csendes A, Korn O, Burdiles P. Historia de la cirugía bariátrica en Chile. Rev Chil Cir 2006; 58: 386-9.

29. Nguyen NT, Paya M, Stevens CM, Mavandadi S, Zainabadi K, Wilson SE. The relationship between hospital volume and outcome in bariatric surgery at academic medical centers. Ann Surg 2004; 240: 586-934.

30. Murr MM, Martin T, Haines K, Torrella T, Dragotti R, Kandil A, et al. A state-wide review of contemporary outcomes of gastric bypass in Florida: does provider volume impact outcomes? Ann Surg 2007; 245: 699-706.

31. Ballantyne GH, Belsley S, Stephens D, Saunders JK, Trivedi A, Ewing DR, et al. Bariatric surgery: low mortality at a high-volume center. Obes Surg 2008; 18: 660-7.

32. Schauer P, Ikramuddin S, Hamad G, Gourash W. The learning curve for laparoscopic Roux-en-Y gastric bypass is 100 cases. Surg Endosc 2003; 17: 212-5.

33. Oliak D, Ballantyne GH, Weber P, Wasielewski A, Davies RJ, Schmidt HJ. Laparoscopic Roux-en-Y gastric bypass: defining the learning curve. Surg Endosc 2003; 17: 405 8.

34. Shikora SA, Kim JJ, Tarnoff ME, Raskin E, Shore R. Laparoscopic Roux-en-Y gastric bypass: results and learning curve of a high-volume academic program. Arch Surg 2005; 140: 362-7.

35. Sovik TT, Aasheim ET, Kristinsson J, Schou CF, Diep LM, Nesbakken A, et al. Establishing laparoscopic Roux-en-Y gastric bypass: perioperative outcome and characteristics of the learning curve. Obes Surg 2009; 19: 158-65.

36. Escalona A, Devaud N, Pérez G, Crovari F, Boza C, Viviani $\mathrm{P}$, et al. Antecolic versus retrocolic alimentary limb in laparoscopic Roux-en-Y gastric bypass: a comparative study. Surg Obes Relat Dis 2007; 3: 423-7.

37. Escalona A. Complicaciones quirúrgicas en bypass gástrico laparoscópico. Rev Chil Cir 2006; 58: 97-105.

38. Escalona A, Devaud N, Boza C, Pérez G, Fernández J, Ibáñez L, et al. Gastrojejunal anastomotic stricture after Roux-en-Y gastric bypass: ambulatory management with the Savary-Gilliard dilator. Surg Endosc 2007; 21: 765-8. 